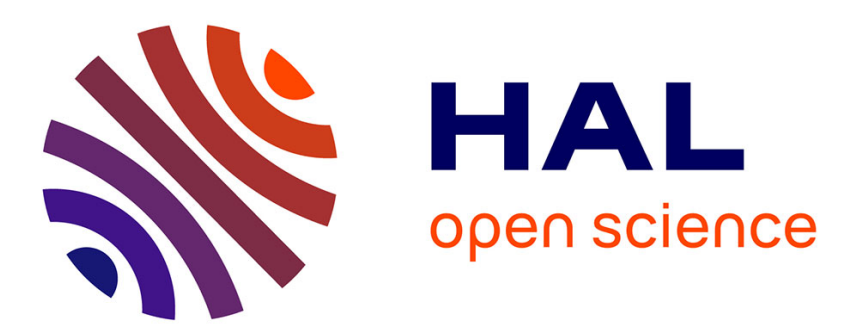

\title{
Size distribution of associated clusters in liquid alcohols: Interpretation of simulation results in the frame of SAFT approach
}

Jiri Janecek, Patrice Paricaud

\section{- To cite this version:}

Jiri Janecek, Patrice Paricaud. Size distribution of associated clusters in liquid alcohols: Interpretation of simulation results in the frame of SAFT approach. Journal of Chemical Physics, 2013, 139, pp.174502. 10.1063/1.4827107 . hal-01058097

\section{HAL Id: hal-01058097 \\ https://hal-ensta-paris.archives-ouvertes.fr/hal-01058097}

Submitted on 26 Aug 2014

HAL is a multi-disciplinary open access archive for the deposit and dissemination of scientific research documents, whether they are published or not. The documents may come from teaching and research institutions in France or abroad, or from public or private research centers.
L'archive ouverte pluridisciplinaire HAL, est destinée au dépôt et à la diffusion de documents scientifiques de niveau recherche, publiés ou non, émanant des établissements d'enseignement et de recherche français ou étrangers, des laboratoires publics ou privés. 


\section{AIP $\begin{gathered}\text { molowanat } \\ \text { chemical Physics }\end{gathered}$}

Size distribution of associated clusters in liquid alcohols: Interpretation of simulation results in the frame of SAFT approach

Jiří Janeček and Patrice Paricaud

Citation: J. Chem. Phys. 139, 174502 (2013); doi: 10.1063/1.4827107

View online: http://dx.doi.org/10.1063/1.4827107

View Table of Contents: http://jcp.aip.org/resource/1/JCPSA6/v139/i17

Published by the AIP Publishing LLC.

Additional information on J. Chem. Phys.

Journal Homepage: http://jcp.aip.org/

Journal Information: http://jcp.aip.org/about/about_the_journal

Top downloads: http://jcp.aip.org/features/most_downloaded

Information for Authors: http://jcp.aip.org/authors

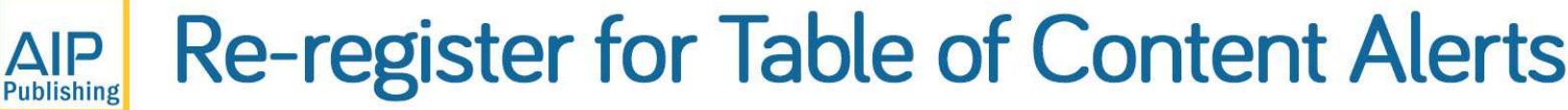

\section{Create a profile.




\title{
Size distribution of associated clusters in liquid alcohols: Interpretation of simulation results in the frame of SAFT approach
}

\author{
Jiří Janeček ${ }^{1,2, a)}$ and Patrice Paricaud ${ }^{1, b)}$ \\ ${ }^{1}$ ENSTA ParisTech, UCP, 828 Boulevard des Maréchaux, 91762 Palaiseau Cedex, France \\ ${ }^{2}$ Mines ParisTech, CTP, 35 rue Saint Honoré, 77305 Fontainebleau Cedex, France
}

(Received 4 July 2013; accepted 14 October 2013; published online 4 November 2013)

\begin{abstract}
The size distribution and topology of associated clusters for primary alcohols is studied using molecular dynamics simulations. Liquid ethanol, propanol, butanol, hexanol, and octanol are simulated at pressure $P=1 \mathrm{bar}$ and temperatures $T=300 \mathrm{~K}, T=350 \mathrm{~K}$, and $T=400 \mathrm{~K}$. The fractions of molecules with different sets of hydrogen bonded partners, the size of associated cluster and the site-site distribution functions between atoms participating on hydrogen bonding are extracted from simulated trajectories. For all alcohols longer than ethanol, the length of the alkyl chain has only a marginal effect on the association. Consequently, related properties like coordination numbers of hydroxyl group, size distribution of associates, or fractions of differently coordinated alcohol molecules are independent on the molecular size. Although we employed a force-field without involved polarizability, we observe a positive cooperativity of hydrogen bonding simply as a consequence of steric and electrostatic interactions. The size and topology of associates is analyzed within the frame of 3B model of statistical association fluid theory. Although this approach enables good thermodynamic description of systems containing associating compounds, several insufficiencies appear in the description at molecular level. @ 2013 AIP Publishing LLC. [http://dx.doi.org/10.1063/1.4827107]
\end{abstract}

\section{INTRODUCTION}

The formation of hydrogen bonds affects the phase behaviour of many various systems. The importance of hydrogen bonding is usually illustrated on the unique properties of water and on the role of hydrogen bonds in biological systems. Monofunctional alcohols, on the other hand, are rather popular as a subject for the theoretical and experimental studies because of simpler structure of the resulting network. ${ }^{1}$

The characteristic frequency of the stretching mode of the $\mathrm{O}-\mathrm{H}$ bond is affected by the local environment which enables to distinguish between hydroxyl groups participating and nonparticipating on hydrogen bonding. Infrared spectroscopy thus represents the most popular experimental methods to study the microscopic structure of the hydrogen bonded systems. $^{2-8}$ The neutron- or X-ray diffraction techniques ${ }^{9-13}$ and the dielectric relaxation ${ }^{14,15}$ can provide further information about the structure and dynamics of the hydrogen bonded systems. Indirect information about the hydrogen bonding can be deduced also from macroscopic properties (e.g., from the viscosity measurements or $P v T$ behaviour and phase equilibrium data). ${ }^{16-20}$

As for the theoretical approaches, the computational demands of $a b$ initio calculations reduce their applicability to relatively small clusters. Nevertheless, these methods significantly contributed to the interpretation of the spectroscopic measurements and to the understanding the mechanism of the cooperativity of the hydrogen bonding. ${ }^{21-24}$ The classi-

\footnotetext{
${ }^{a)}$ Email: janecek_jiri@centrum.cz

${ }^{b)}$ Email: patrice.paricaud@ensta-paristech.fr
}

cal atomistic simulations can treat much larger systems (compared to quantum methods) and thus they represent important tool to study the internal structure of associating systems. Several recent as well as classical studies were aimed to analyze the size and topology of associated species. ${ }^{11,25-33}$

The thermodynamic description of systems containing associating compounds represents a difficult task, especially in the case of mixtures of associating and non-associating components (e.g., alcohol-alcane) or systems which experience the cross-association despite the particular components alone do not associate (e.g., chloroform-acetone). ${ }^{34}$ Among several approaches suggested to treat the behaviour of associating systems, the statistical association fluid theory (SAFT) of Wertheim ${ }^{35-38}$ gained a prominent position and was incorporated into a family of equations of state. ${ }^{39-43}$

Within the SAFT approach, the associating molecules are represented as some geometric bodies (sphere, flexible chain, convex body) which contain at their surface small sites interacting with (at most one) other site on neighbouring molecules via square-well attraction. The contribution to the Helmholtz energy (and other thermodynamic properties) due to the association is then expressed as a simple function of the fractions of associating sites which are not bonded to any partner. Despite good agreement was found between the SAFT approach and computer simulations data (for respective model of molecules, i.e., with square-well associating sites) ${ }^{4-46}$ there are two main issues in which the theory departs from the reality: (i) the theory assumes formation of open tree-like associates only (without the possibility to form cyclic structures) and (ii) the hydrogen bonding is modelled by square-well interaction and the effect of the electrostatic (dipolar) interactions is 
neglected. Both issues were analyzed by Sear and Jackson $^{47-49}$ but increased complexity of suggested solutions prevented from their wide use in thermodynamic models for real fluid systems.

The monofunctional alcohols are modelled either using a model containing two sites, one with the acceptor- and the second with donor-function (this model is denoted as 2B model) or by a model with one donor- and two acceptor-sites (3B model). The 3B model might seem - at first sight - to have better molecular background since the two lone electron pairs at oxygen atom can accept two hydrogens from neighbouring molecules. However, in pure alcohols there is a deficit of donor sites (hydrogen atoms) and the second acceptor site has lower chance to form hydrogen bond and that is why the performance of both models is comparable. The importance of appropriate association scheme was extensively discussed only in the case of carboxylic acids which oscillate between 2B model and 1A model (in which molecules contain only a single site which is a model adequate for diluted systems in which the carboxylic groups form cyclic dimers). ${ }^{50-52}$

Biomass based products are more and more used as substituent of petroleum fluids, both as fuels and as raw materials for the chemical industry. ${ }^{53}$ The bio-oils are complex mixtures with a high content of oxygenated compounds containing mainly hydroxyl, carbonyl, ether, and carboxyl groups, i.e., groups which can participate on hydrogen bonding. Also the carbon capture using aminoalcohols represents a perspective area in which the association is involved. ${ }^{54,55}$ In both these examples, the correct treatment of the association is crucial to obtain good thermodynamic description. In the first case because the bio-oils are mixtures of acceptor rich organic compound (esters, ketones) with water. In case of alkanolamines, combination of donor-rich amino groups $-\mathrm{NH}_{2}$ and acceptorrich hydroxyls at one molecule can result into formation of $3 \mathrm{D}$ network.

The need for an adequate description of such complex systems leads to development of group contribution versions of SAFT-based equations of state, ${ }^{56-62}$ which represents an analogy of the standardized force-fields used in molecular simulations; the parameters describing the association are then considered as universal parameters for a whole class of compounds (usually with the exception of the first members of homological series). Recently, Ferrando et al. ${ }^{63}$ employed Monte Carlo simulations to obtain the fractions of molecules that do not participate in hydrogen bonding and used these values together with experimental coexistence data to determine the pure component parameters of Polar PerturbedChain SAFT equation of state.

In this work, we analyze the size and structure of hydrogen bonded clusters of molecules obtained by molecular dynamics simulations. The observations are interpreted in the view of statistical association fluid theory and the approximations commonly done within this approach. One of the aims of this work is to compare the quality of the two most frequently employed association schemes used for monofunctional alcohols not only from the view of thermodynamic description but also from the microscopic (structural) point of view. Another goal is to find out whether the assumption of the universality of the association parameters adequate.

\section{TECHNICAL DETAILS}

We performed set of molecular dynamics simulations of five primary non-branched alcohols - ethanol, 1-propanol, 1butanol, 1-hexanol, and 1-octanol; in the following text we are going to use the names without numerical locants. The simulations were carried out using the molecular dynamics module of the TINKER package ${ }^{64}$ at NPT-like conditions. The equations of motion were integrated using the Beeman algorithm with the time step $\Delta t=1 \mathrm{ft}$. The pressure and temperature control was done using the bath coupling method of Berendsen et al.; ${ }^{65}$ the default values of coupling times for pressure $\tau_{p}=2 \mathrm{ps}$ and for temperature $\tau_{T}=0.1 \mathrm{ps}$ were used. The length of the equilibration phase was typically $1 \mathrm{~ns}$, the length of each production run was $5 \mathrm{~ns}$. The coordinates of all atoms were stored after every 1000th step for the structural analysis. The intermolecular interactions were modelled using the All Atom version of Optimized Potential for Liquid Simulations (OPLS-AA) force field. ${ }^{66}$ The electrostatic interactions were treated by the smooth particle mesh Ewald technique as implemented in the TINKER package. The Lennard-Jones interactions were truncated at $R_{c}=9 \AA$ without adding any long range corrections. Every system was simulated under pressure $P=1 \mathrm{bar}$ at three different temperatures, $T=300 \mathrm{~K}, T$ $=350 \mathrm{~K}$, and $T=400 \mathrm{~K}$. The number of molecules in the simulation box was 330 for ethanol, 270 for propanol, 210 for butanol, 165 for hexanol and 130 in the case of octanol. Simulation runs with twice higher number of molecules were done for ethanol, butanol, and octanol to estimate the influence of finite size effects. These simulations were done with united-atoms OPLS force-field. ${ }^{25}$

We used the Luzar-Chandler criterion ${ }^{67}$ to distinguish hydrogen bonded molecules - two molecules are bonded, if the separation between the hydrogen atom of the donor molecules and the oxygen atom of acceptor molecule are closer than the some minimal separation $r_{\min }$ and the angle $\alpha_{\mathrm{OH} \ldots \mathrm{O}}$ is larger than $150^{\circ}$. The minimal distance was taken to be temperature dependent, equal to the distance of the first minimum on the site-site distribution function between the oxygen atoms of the hydroxyl groups. It should be noted that the influence of temperature dependence of the distance cutoff is negligible. This criterion can ensure that not more than two donor molecules can be bonded to one acceptor molecule.

Using the configurations saved during the production run, we have calculated the number and type of partners bonded through hydrogen bonds. With respect to the employed HB criterion, six different bonding states are possible; the fractions of molecules in different bonding states can be represented by a $3 \times 2$ matrix $\xi$, for the elements of which we use notation $\xi_{i_{d}, j_{a}}$ where $i_{d}=(0,1$, or 2$)$ is the number of donor molecules bonded to the molecules and $j_{a}=(0$ or 1$)$ is the number of its acceptors. The relation between $\xi_{i_{d}, i_{a}}$ and the fraction of molecules with $m \mathrm{H}$-bonds $f_{m}$ (which is used in classical works $\left.{ }^{27,68}\right)$ is straightforward: $f_{m}=\sum_{i_{d}^{*}, j_{a}^{*}} \xi_{i_{d}, j_{a}}$, where the asterisks denote that the summation runs over all pairs which satisfy $i_{d}+j_{a}=m$. 
In Figure 1, we show a schematic picture of a molecule with one donor- (red circle) and two acceptor- (blue circles) sites. Three different tetramers composed of such associating molecules are also shown (the green circles denote existing hydrogen bonds). The greek letters correspond to the notation used by Sillrén et al. ${ }^{33}-\alpha$ denotes the molecules not participating on hydrogen bond formation, $\beta$ denotes the "root" molecules of particular clusters (the molecules with nonbonded hydrogen site), $\gamma$ stands for "leafs" (end molecules of particular branches) and $\delta$ denotes the internal groups. The subscripts in case of roots and internal groups denote the number of bonded donors, so roots of true trees $\beta_{1}$ are distinguished from U-like associates $\beta_{2}$ and the standard internal groups $\delta_{1}$ from branching nodes $\delta_{2}$. In the matrix notation described in previous paragraph, the free monomers $\alpha$ correspond to $\xi_{0,0}$ component, tree roots $\beta_{1}$ to $\xi_{1,0}$, roots with branching $\beta_{2}$ to $\xi_{2,0}$, leafs $\gamma$ to $\xi_{0,1}$, and the normal and branching internal groups $\delta_{1}$ and $\delta_{2}$ to $\xi_{1,1}$ and $\xi_{2,1}$ components.

The cluster-size distributions discussed in this work correspond to "fraction of clusters of size $n$ from the total amount of clusters"; in agreement with Sillrén et al. ${ }^{33}$ we use for it symbol $r(n)$. The size of clusters is considered irrespective of their topology, thus all tetramers shown in Figure 1 contribute to the same value, $n=4$. Thanks to only one donor site (hydrogen atom) per molecule, each associate contains either one non-bonded hydrogen site (root molecule) or just one cyclic structure. In order to simplify the analysis, if any cyclic substructure is detected, all hydrogen bonds leading to the cycle are cancelled and the resulting substructures are considered as separate n-mers. Example of such decomposition is shown in Figure 2, where a 12-mer is divided into one cyclic and one branched hexamers. This simplification leads to a small underestimation of the fraction of larger associates, however with respect to low fraction of molecules bonded in the cyclic substructures, the effect of this approximation is not crucial.

\section{RESULTS AND DISCUSSION}

The simulated densities at $P=1$ bar for the five alcohols are compared with the experimental saturated liquid

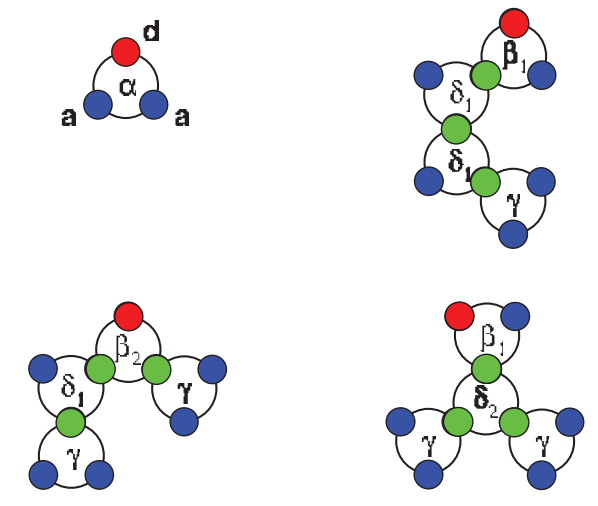

FIG. 1. Examples of alcohol molecules in different bonding states. Six different states are possible - non-bonded molecules $(\alpha)$, two different types of roots $\left(\beta_{1}\right.$ and $\beta_{2}$ ), end-groups of branches (leafs; $\gamma$ ), and two types of internal groups $\left(\delta_{1}\right.$ and $\left.\delta_{2}\right)$. The red circles represent non-bonded hydrogens, the blue circles the lone electron pairs, and the green circles denote the existing hydrogen bonds.
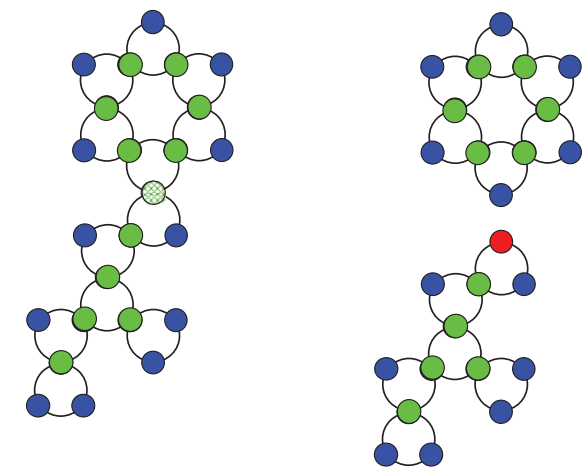

FIG. 2. An example of the decomposition of a lasso-like associate into the cyclic and acyclic parts. While the original lasso-like structure does not contain any root molecule (molecule with non-bonded hydrogen site), the parted branch contains just one such molecule.

densities $^{69}$ in Figure 3. Although the OPLS-AA force field parameters were optimized to reproduce the liquid coexistence density at standard temperature (together with the value of the vaporization enthalpy), we observe significant differences, most evident for the shorter alcohols and at higher temperatures. This disagreement follows from neglecting the Lennard-Jones interactions beyond the cut-off distance, $R_{c}=9 \AA$, and is comparable with the difference observed for nonpolar fluids. ${ }^{70-72}$

The course of site-site distribution functions between the oxygen atoms (upper part) and between oxygen and hydrogen atoms (lower part) of the hydroxyl groups is shown in Figure 4 for different alcohols at temperature $T=300 \mathrm{~K}$. For the sake of clarity, only the curves for ethanol, butanol, and octanol are plotted. The thin (increasing) lines of corresponding type and colour show the average number of sites of type $B$ up to given separation $r$ from site $A$,

$$
N_{B}(r)=\rho_{B} \int_{0}^{r} 4 \pi x^{2} g_{A-B}(x) d x .
$$

The value of this function at the distance of the first minimum on the rdf corresponds to the coordination number, i.e., to the average number of atoms (or groups) in the first

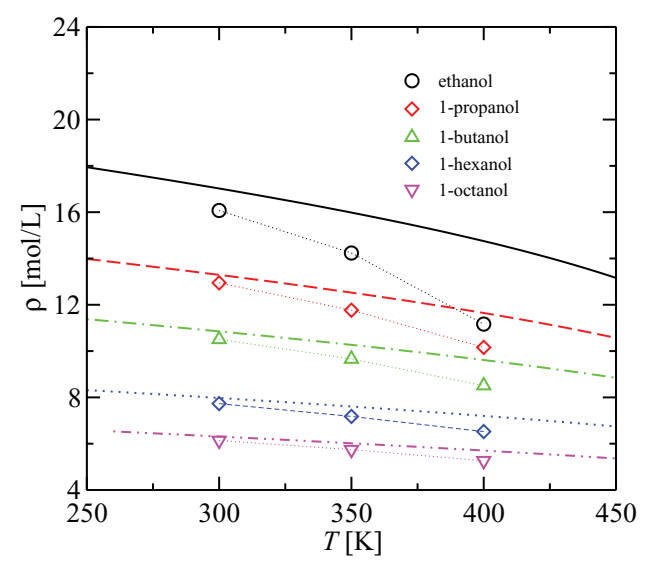

FIG. 3. The comparison of the simulated liquid densities (symbols) with the experimental values ${ }^{69}$ (lines). The symbols represent different alcohols according to the inserted legend, the experimental values are lines of corresponding colour (and order from top to down). 


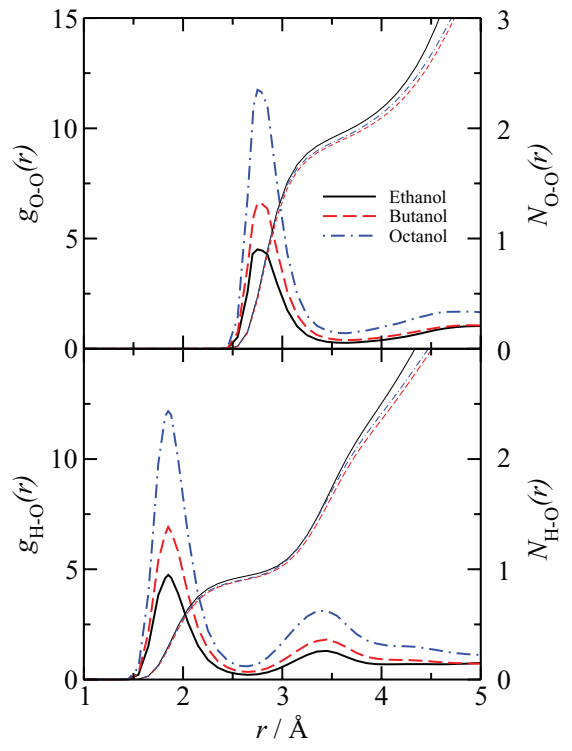

FIG. 4. The oxygen-oxygen (upper part) and oxygen-hydrogen (lower part) radial distribution functions for ethanol (solid black lines), butanol (dashed, red), and octanol (dashed-dotted, blue) at $T=300 \mathrm{~K}$. The thin lines show the course of coordination numbers; for these curves the right vertical axis with different scale is used.

solvation layer. For the three presented alcohols we found approximately one oxygen atom in the closest vicinity of hydrogen of hydroxyl group $N_{\mathrm{H}-\mathrm{O}}=0.94 / 0.91 / 0.92$ (ethanol/ butanol/octanol) and almost two closest hydroxyl groups around the hydroxyl oxygen $\left(N_{\mathrm{O}-\mathrm{O}}=1.98 / 1.91 / 1.93\right)$. The values of coordination numbers as well as the positions of the first maxima and consequent minima on both distribution functions are almost independent on the length of the alcohol. This indicates the equable tendency to form the hydrogen bonds irrespective of the density of hydroxyl groups in particular systems. Different heights of the first peak are caused by normalization of rdfs to the number density of hydroxyl groups (which is smaller for longer alcohols).

In Figure 5, the same site-site rdfs are shown for ethanol at three different temperatures. Expectably, the temperature increase leads to the decay of the coordination numbers the number of closest oxygens to a hydrogen site decreases as $N_{\mathrm{H}-\mathrm{O}}=0.94 / 0.84 / 0.68$ and that for oxygen sites as $N_{\mathrm{O}-\mathrm{O}}$ $=1.98 / 1.82 / 1.52$. The position of the first peak and of the first minimum on both distribution functions is slightly increased with increasing temperature; that is why we employ temperature dependent value of the threshold distance in the hydrogen bond criterion $-r_{\text {min }}=2.650 \AA$ for $T=300 \mathrm{~K}, r_{\text {min }}$ $=2.675 \AA$ for $T=350 \mathrm{~K}$, and $r_{\text {min }}=2.70 \AA$ for $T=400 \mathrm{~K}$. For higher alcohols (not shown in Figure 5), the coordination numbers are slightly lower than those for ethanol. The position of first minima and maxima on rdfs is the same as in the case of ethanol.

The average sizes of associated clusters, $\langle n\rangle$, and the variances, $\left\langle n^{2}\right\rangle-\langle n\rangle^{2}$, for all simulated systems are listed in Table I. Expectably, with increasing temperature the average cluster size as well as the width of the size distribution become smaller because the degree of association decreases with increasing temperature (the hydrogen bonds dissoci-

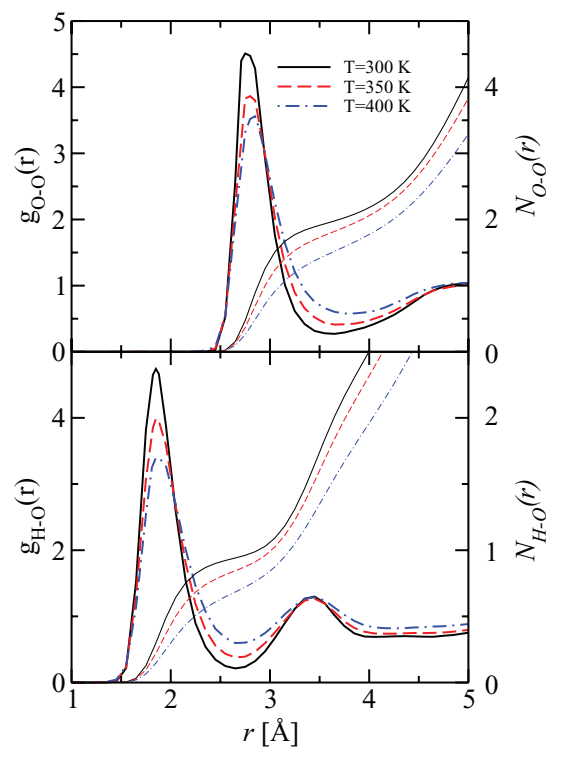

FIG. 5. The oxygen-oxygen (upper part) and oxygen-hydrogen (lower part) radial distribution functions for ethanol at different temperatures. The thin lines show the course of coordination numbers.

ate). The temperature trends of both characteristics are in agreement with the neutron diffraction data (respectively, Empirical Potential Structure Refinement based on neutron diffraction). ${ }^{13}$ The mean values at $T=300 \mathrm{~K}$ for propanol and butanol are approximately $25 \%$ higher compared with the values of Ref. 33.

Although the distribution characteristics presented in Table I are not convincing, one can again take notice of rather close values for the longer alcohols. Better evidence for low influence of chain length on the HB formation brings Figure 6, which shows the cluster-size distributions for all simulated alcohols. The course of cluster-size distribution functions is only marginally affected by the number of carbon atoms in the chain. Again, ethanol shows higher tendency to associate and the relative amount of larger clusters is higher.

In order to simplify the cluster-size analysis, we decomposed aggregates containing cyclic structure into the ring and the branches and these fragments were treated as separate aggregates. The rings typically are composed of 3-6 molecules. At $T=300 \mathrm{~K}$, they contain up to $2 \%$ of the total amount of molecules and less than $1 \%$ at higher temperatures. The size-distribution of cycles is again independent of the type of

TABLE I. The average cluster sizes and its variances for the simulated alcohols at different temperatures.

\begin{tabular}{lccccc}
\hline \hline$T(\mathrm{~K})$ & Ethanol & Propanol & Butanol & Hexanol & Octanol \\
\hline & & & $\langle n\rangle$ & & \\
300 & 4.90 & 3.78 & 3.97 & 3.82 & 3.90 \\
350 & 2.98 & 2.30 & 2.40 & 2.30 & 2.32 \\
400 & 1.98 & 1.67 & 1.67 & 1.64 & 1.61 \\
& & & $\left\langle n^{2}\right\rangle-\langle n\rangle^{2}$ & & \\
300 & 31.9 & 14.9 & 16.9 & 13.5 & 15.8 \\
350 & 8.99 & 3.94 & 4.47 & 3.85 & 4.11 \\
400 & 2.71 & 1.41 & 1.44 & 1.34 & 1.26 \\
\hline \hline
\end{tabular}




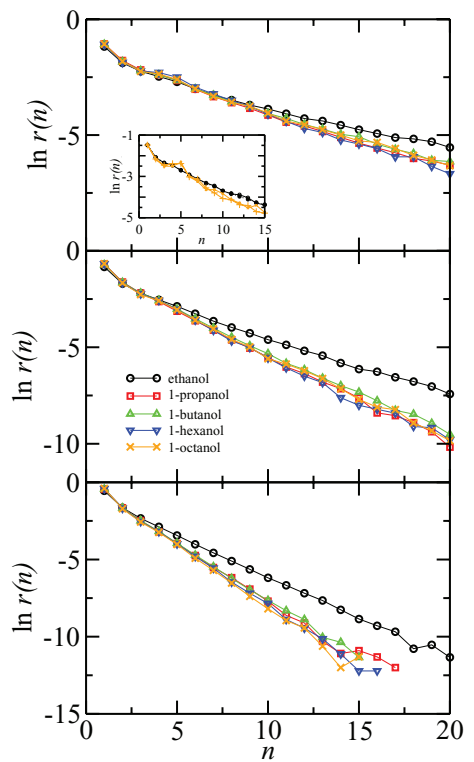

FIG. 6. The distribution of cluster-sizes for all simulated alcohols at different temperatures. The upper figure shows the results for $T=300 \mathrm{~K}$, the middle one for $T=350 \mathrm{~K}$, and the lower for $T=400 \mathrm{~K}$. The inserted graph in the upper plot shows the comparison at $T=300 \mathrm{~K}$ for ethanol simulated with 330 molecules (open circles) and with 660 molecules (solid circles) and for octanol with 130 (x-symbols) and 260 (+ symbols).

alcohol. The decomposition causes some underestimation of fractions of larger clusters and overvaluation of shorter ones (mainly those with $n=3-6$ ); however, with respect to low number of molecules in cyclic structures this effect can be in first view neglected.

The inset figure in the topmost panel of Figure 6 compares the cluster size distributions at $T=300 \mathrm{~K}$ obtained with 330 ethanol molecules or 130 octanol molecules with those obtained in twice larger systems (this simulations were done with united-atom OPLS force-field, that is why the course of distribution functions is different from those shown in the main plot). This figure shows that the original systems are large enough and the calculated properties of HB networks are not influenced by the finite size effects. With respect to rather low number of molecules, this fact might seem to be surprising. However, it is in accordance with our previous findings for thermodynamic and structural properties of Lennard-Jones fluid that at liquid densities the implicit finite size effects become negligible if the box size is higher than ten-times the particle diameter. ${ }^{73,74}$

The cluster-size distribution function for ethanol at $T=300 \mathrm{~K}$ is shown in Figure 7. The simulated distribution (crosses) is compared with three models $-2 \mathrm{~B}$ association scheme of SAFT (solid black line), 3B scheme (dashed red line), and with the model suggested recently by Sillrén et al. $^{33}$ (dashed-dotted blue line). Within the last model, the fraction of clusters of size $n$ is given by recurrent formula

$$
r(n)=p_{a}\left(1-p_{b}\right) r(n-1)+p_{a} p_{b} \sum_{k=1}^{k=n-2} r(k) r(n-k-1),
$$

where parameter $p_{a}$ represents the probability of accepting a first hydrogen bond from a neighboring molecule and $p_{b}$ is

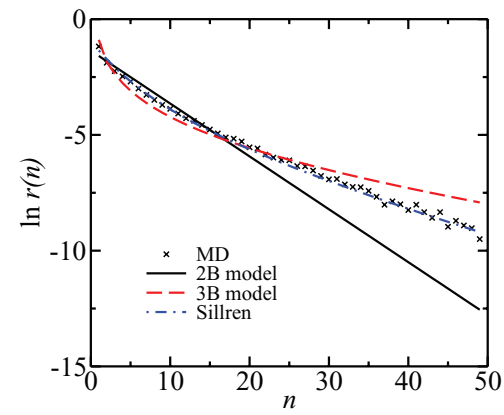

FIG. 7. The comparison of simulated cluster-size distribution for ethanol at $T=300 \mathrm{~K}$ (symbols) with the 2B model (solid black line), 3B model (dashed red line) and with the two-probability tree model (dashed-dotted blue line).

the conditional probability of forming a second bond given that a first bond already exists. The first term in the series, the fraction of monomers, is equal to $r(1)=1-p_{a}$. The $2 \mathrm{~B}$ model of SAFT represents a limiting case of this model with $p_{b}=0$ and the distribution becomes geometric (in polymer chemistry called Anderson-Schulz-Flory distribution),

$$
r(n)=p_{a}^{n-1}\left(1-p_{a}\right) .
$$

The 3B model of SAFT represents also a special case of (2) which assumes $p_{b}=p_{a}^{2}$. This condition follows from the definition of model in which it is assumed that the formation of hydrogen bonds on one association site does not affect the association of the remaining parts of the molecule.

The model distributions shown in Fig. 7 are calculated to reproduce the mean size of associated cluster, in case of the general (two-parameter) form also the variance. Clearly, the more flexible two-parameters Sillrén distribution (Eq. (2)) can fit the simulated distribution better than the two special cases. In accordance with Ref. 33, the fraction of short associates is slightly overestimated by this model (except the monomers). The true distribution curve lies between the two limiting cases for 2B- and 3B-model. Thus, the associates in liquid alcohols are formed not solely by linear chains (as it is assumed by the 2B model), however the importance of branching predicted by $3 \mathrm{~B}$ model is overvalued.

The probability parameters for the three different association models are summarized in Table II. For all three models the growing probability decreases with increasing temperature, in accordance with the trend for the mean cluster sizes. The approximations for the branching probabilities within the 3B model, $p_{b}=p_{a}^{2}$, are significantly higher compared to the pseudoexperimental values. Moreover, with increasing temperature the true branching probabilities increase, while those for 3B model decrease.

The fitted values of $p_{a}$ and $p_{b}$ for propanol and butanol at $T=300 \mathrm{~K}$ are slightly higher compared to the values reported in Ref. $33-p_{a} / p_{b}=0.642 / 0.042^{33}$ vs. $p_{a} / p_{b}=0.695 / 0.058$ (this work) for propanol and $p_{a} / p_{b}=0.633 / 0.033^{33}$ vs. $p_{a} / p_{b}$ $=0.708 / 0.057$ (this work). The temperature trends of our values are in agreement with Ref. 13. We note that even when we employed exactly the same criterion for hydrogen bonding $(\mathrm{O} \cdots \mathrm{H}$ distance between 1.4 and $2.4 \AA$ without any angular limitations), considerable differences were observed between our values and those of Ref. 33. The most important factor is 
TABLE II. The probability parameters describing the association of alcohols for the 2B and 3B models of SAFT and for the tree model of Ref. 33.

\begin{tabular}{lccccc}
\hline \hline$T(\mathrm{~K})$ & Ethanol & Propanol & Butanol & Hexanol & Octanol \\
\hline & & \multicolumn{5}{c}{ 2B model: $p_{a}$} \\
300 & 0.796 & 0.735 & 0.748 & 0.738 & 0.744 \\
350 & 0.664 & 0.565 & 0.584 & 0.565 & 0.568 \\
400 & 0.495 & 0.400 & 0.402 & 0.390 & 0.378 \\
& & & 3B model: $p_{a}$ & & \\
300 & 0.590 & 0.560 & 0.566 & 0.561 & 0.564 \\
350 & 0.522 & 0.465 & 0.476 & 0.465 & 0.466 \\
400 & 0.420 & 0.355 & 0.357 & 0.348 & 0.339 \\
& & & Tree model: $p_{a}$ & & \\
300 & 0.741 & 0.695 & 0.708 & 0.714 & 0.706 \\
350 & 0.605 & 0.526 & 0.545 & 0.530 & 0.525 \\
400 & 0.445 & 0.367 & 0.369 & 0.356 & 0.344 \\
& & & Tree model: $p_{b}$ & & \\
300 & 0.0735 & 0.0579 & 0.0572 & 0.0346 & 0.0534 \\
350 & 0.0974 & 0.0746 & 0.0723 & 0.0671 & 0.0820 \\
400 & 0.1124 & 0.0899 & 0.0906 & 0.0930 & 0.0981 \\
\hline \hline
\end{tabular}

probably different density of simulated systems (Sillrén et al. perform MC simulations in $N V T$ ensemble, probably at the experimental coexistence density which is $3 \%$ higher compared to our density).

The basic quantity which enables to express the association contribution to thermodynamic properties within the SAFT is usually called "fraction of non-bonded sites," more precisely fraction of molecules with bonding site of type $A$ non-bonded, $X_{A}$. From now on, we leave the 2B model (which does not count for branching) and the two-parameter model of Sillrén et al. (which is not compatible with the classical SAFT approach) and in the following considerations we concern only the $3 \mathrm{~B}$ model and simulation results.

We are going to follow the notation of Huang and Radosz $^{39}$ and to denote the two acceptor sites as $A$ and $B$ and the single donor site as $C$. Since the fraction of bonded donor sites is equal to the sum of fractions of the two (formally) different acceptor sites $\left(\left(1-X_{C}\right)=\left(1-X_{A}\right)+\left(1-X_{B}\right)\right)$, the three fractions of non-bonded sites satisfy

$$
X_{A}+X_{B}-X_{C}=1 .
$$

Thanks to the equivalence of the two acceptor sites, $X_{A}=X_{B}$, there is a unique relation between the fractions of non-bonded donors and acceptors,

$$
X_{C}=2 X_{A}-1 .
$$

Thus, for one-component systems only one of the fractions is sufficient. We notice that while the fraction of non-bonded donor sites $X_{C}$ is zero in the case of complete association, those for the two distinguished acceptors have limiting value of $X_{A}=X_{B}=0.5$.

The values of fractions of non-bonded sites $X_{A}$ and $X_{C}$ can be calculated from the fractions of molecules with different number of hydrogen bond partners which are listed in Table III. Namely, the fraction of molecules with free H-site $X_{C}$ is simply the sum of the three contributions,

$$
X_{C}=\xi_{0,0}+\xi_{1,0}+\xi_{2,0}
$$

TABLE III. The fractions of molecules according to number of different HB partners for different alcohols at different temperatures. For all three temper-

\begin{tabular}{|c|c|c|c|c|c|}
\hline & Ethanol & Propanol & Butanol & Hexanol & Octanol \\
\hline & & & $T=300 \mathrm{~K}$ & & \\
\hline \multirow[t]{2}{*}{$\xi_{0,0}$} & 6.2 & 9.1 & 8.6 & 8.8 & 8.8 \\
\hline & (7.1) & (10.3) & $(9.9)$ & (9.8) & (9.7) \\
\hline \multirow[t]{2}{*}{$\xi_{1,0}$} & 12.8 & 16.2 & $15.5)$ & 15.7 & 15.4 \\
\hline & $(9.5)$ & (12.1) & (11.6) & (11.8) & (11.6) \\
\hline \multirow[t]{2}{*}{$\xi_{2,0}$} & 0.77 & 0.63 & 0.57 & 0.61 & 0.58 \\
\hline & (3.1) & (3.6) & (3.5) & (3.5) & (3.5) \\
\hline \multirow[t]{2}{*}{$\xi_{0,1}$} & 19.1 & 19.9 & 19.1) & 19.2 & 19.2 \\
\hline & $(28.8)$ & (29.4) & $(29.3)$ & $(29.3)$ & $(29.3)$ \\
\hline \multirow[t]{2}{*}{$\xi_{1,1}$} & 56.4 & 51.7 & 53.8 & 53.4 & 53.5 \\
\hline & $(38.5)$ & (34.5) & $(35.4)$ & $(35.1)$ & $(35.3)$ \\
\hline \multirow[t]{3}{*}{$\xi_{2,1}$} & 4.7 & 2.5 & 2.4 & 2.3 & 2.6 \\
\hline & (12.9) & (10.1) & (10.7) & $(10.5)$ & $(10.6)$ \\
\hline & & & $T=350 \mathrm{~K}$ & & \\
\hline$\xi_{0,0}$ & 14.5 & 21.8 & 20.2 & 22.0 & 22.1 \\
\hline$\xi_{1,0}$ & 17.9 & 20.7 & 20.3 & 20.3 & 20.0 \\
\hline$\xi_{2,0}$ & 0.90 & 0.67 & 0.64 & 0.66 & 0.60 \\
\hline$\xi_{0,1}$ & 22.7 & 23.4 & 23.0 & 22.9 & 22.5 \\
\hline$\xi_{1,1}$ & 41.0 & 32.1 & 34.4 & 32.9 & 33.5 \\
\hline \multirow[t]{2}{*}{$\xi_{2,1}$} & 3.0 & 1.3 & 1.4 & 1.3 & 1.3 \\
\hline & & & $T=400 \mathrm{~K}$ & & \\
\hline$\xi_{0,0}$ & 29.1 & 38.7 & 38.5 & 40.1 & 41.7 \\
\hline$\xi_{1,0}$ & 20.5 & 20.7 & 20.6 & 20.2 & 19.8 \\
\hline$\xi_{2,0}$ & 0.77 & 0.51 & 0.51 & 0.50 & 0.49 \\
\hline$\xi_{0,1}$ & 23.4 & 22.3 & 22.2 & 21.8 & 21.3 \\
\hline$\xi_{1,1}$ & 24.8 & 17.3 & 17.6 & 16.8 & 16.2 \\
\hline$\xi_{2,1}$ & 1.4 & 0.56 & 0.59 & 0.55 & 0.55 \\
\hline
\end{tabular}
atures the values from molecular simulations are shown; for $T=300 \mathrm{~K}$ also the estimates within the 3B model of SAFT are given in parentheses.

and the fraction of non-bonded acceptors is determined as

$$
X_{A}=X_{B}=\xi_{0,0}+\xi_{0,1}+\left(\xi_{1,0}+\xi_{1,1}\right) / 2,
$$

where the factor $1 / 2$ reflects the fact that the acceptor sites $A$ and B are distinguished in the data of Table III ( $\xi_{1,1}$ represents the fraction of molecules bonded on sites $C$ and $B$ as well as the fraction of molecules bonded on sites $C$ and $A$ ).

The fractions $X_{A}$ and $X_{C}$ observed in MD simulations are listed in Table IV; the numbers in parentheses are the values of $X_{A}$ calculated from the growing probabilities $p_{a}$ for 3B model using the relation for the fraction of monomers,

$$
\xi_{0,0}=\frac{\left(1-p_{a}\right)}{\langle n\rangle}=X_{A}^{2}\left(2 X_{A}-1\right) .
$$

The difference between the values counted directly from simulation data and those calculated from $p_{a}$ (which is extracted from the average value) is of similar order as reported in Ref. 33.

One of the basic assumptions of the SAFT model is independence of bonding on different sites of one molecule. The elements of matrix $\xi$ can be re-calculated from fractions of non-bonded sites $X_{A}, X_{B}$, or $X_{C}$ for hypothetic situation in which the system behaves like 3B model: $\xi_{0,0}=X_{A} X_{B} X_{C}$, $\xi_{0,1}=X_{A} X_{B}\left(1-X_{C}\right), \ldots, \xi_{2,1}=\left(1-X_{A}\right)\left(1-X_{B}\right)\left(1-X_{C}\right)$. The values of $\xi_{i_{d}, j_{a}}$ calculated in this way from the fraction of molecules with non-bonded hydrogen are shown for 
TABLE IV. The fractions of non-bonded donors $X_{C}$, acceptors $X_{A}$ and free molecules $X_{0}$ as obtained from molecular dynamics simulations. The values of $X_{A}$ in parentheses were calculated from the average value of associated cluster.

\begin{tabular}{lccccc}
\hline \hline & Ethanol & Propanol & Butanol & Hexanol & Octanol \\
\hline & & & $X_{c}$ & & \\
$T=300 \mathrm{~K}$ & 0.198 & 0.260 & 0.247 & 0.251 & 0.248 \\
$T=350 \mathrm{~K}$ & 0.333 & 0.432 & 0.412 & 0.430 & 0.426 \\
$T=400 \mathrm{~K}$ & 0.504 & 0.599 & 0.596 & 0.609 & 0.620 \\
& & & $X_{A}$ & & \\
$T=300 \mathrm{~K}$ & 0.599 & 0.630 & 0.623 & 0.625 & 0.624 \\
& $(0.612)$ & $(0.642)$ & $(0.635)$ & $(0.640)$ & $(0.637)$ \\
$T=350 \mathrm{~K}$ & 0.666 & 0.716 & 0.706 & 0.715 & 0.713 \\
& $(0.676)$ & $(0.723)$ & $(0.714)$ & $(0.723)$ & $(0.721)$ \\
$T=400 \mathrm{~K}$ & 0.752 & 0.800 & 0.798 & 0.804 & 0.810 \\
& $(0.756)$ & $(0.802)$ & $(0.800)$ & $(0.806)$ & $(0.812)$ \\
\hline \hline
\end{tabular}

$T=300 \mathrm{~K}$ in Table III as the numbers in parentheses. In agreement with the comparison of fitted values of $p_{b}$ to simulation data and of those assumed for the $3 \mathrm{~B}$ model $\left(p_{b}=p_{a}^{2}\right)$, we see that the $3 \mathrm{~B}$ model strongly overestimates the amount of branching points - roughly $4 \times$ in case of true branching nodes $\left(\xi_{2,1}\right)$ and almost $6 \times$ in case branching roots $\left(\xi_{2,0}\right)$. As for the monomers, their true concentrations well correspond to the values calculated from $X_{A}$ within the $3 \mathrm{~B}$ model; this is in agreement with the observations of Ferrando et al. ${ }^{63}$

The overvaluation of branching points within the $3 \mathrm{~B}$ model is an indirect consequence of the cooperativity of hydrogen bonding. Direct evidence of the bond cooperativity is the overestimation of the content of short oligomers (dimers pentamers) by the two-parameter model which was observed and discussed by Sillrén et al $^{33}$ ). In Figure 6, this effect is also manifested by change of the slope between $n=2$ and $n=4-5$ (apparent only for $T=300 \mathrm{~K}$ ). In Figure 8, a schematic picture of two different tetramers is shown. In the case of linear associate (left picture), the interaction between $i$ th and $(i+2)$ th molecules is more or less attractive thanks to the suitable orientation of dipoles of hydroxyl groups. Thus, when the third (fourth...) molecule is bonded to a cluster of two molecules, the energetic gain is larger compared to the associate formed by two molecules only. As for the branched structures, the dipoles of the two donor molecules are oriented in energetically unfavourable manner and the occurrence of such configurations is lowered. Since the molecules are in di-
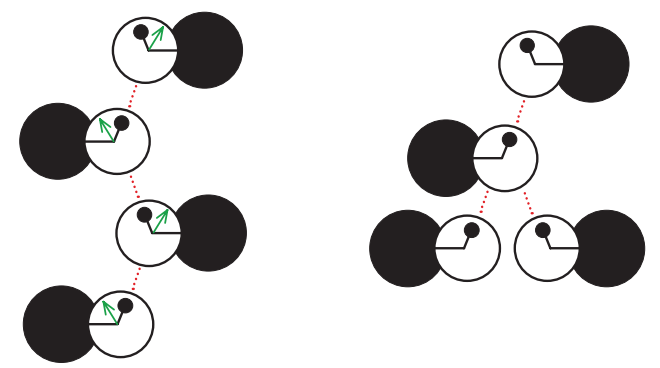

FIG. 8. The schematic picture of linear (left) and branched (right) tetramers. The green arrows in the left part show approximately the orientation of the dipole of the hydroxyl group.

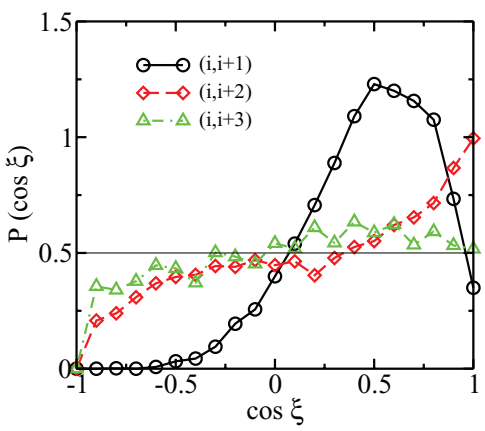

FIG. 9. The probability density for observing the cosine of angle between the dipolar axes for two directly hydrogen bonded molecules (solid black curve + circles), molecules separated by one molecule (dashed red curve + diamonds), and molecules separated by two other molecules in associated cluster (dashed-dotted green curve + triangles).

rect contacts, we should look at the dipoles of the $\mathrm{OH}$ groups instead of the dipole of the whole molecule. We notice that in our simulations only marginal part of the polarizability is included through the flexibility of $\mathrm{OH}$ bond; the induction-like increase of dipole can lead to even stronger effect.

The preferential orientation of HB molecules can be deduced also from Figure 9 where the distribution of the cosine of the angle between the $\mathrm{OH}$ bonds, $\cos \xi$, is shown for pairs of directly bonded molecules (circles) and pairs separated by one- (diamonds) and by two (triangles) molecules for ethanol at $T=300 \mathrm{~K}$. In all three cases, the positive values of $\cos \xi$ are more populated compared to the negative values, i.e., the $\mathrm{OH}$ bonds tend to be oriented in parallel way.

Within the SAFT approach, the fractions of non-bonded sites are calculated from a set of equations. For $3 \mathrm{~B}$ model these equations can be written as

$$
\begin{aligned}
& X_{A}=\left(1+\rho \Delta_{A B} X_{B}+\rho \Delta_{A C} X_{C}\right)^{-1}, \\
& X_{B}=\left(1+\rho \Delta_{A B} X_{A}+\rho \Delta_{B C} X_{C}\right)^{-1}, \\
& X_{C}=\left(1+\rho \Delta_{A C} X_{A}+\rho \Delta_{B C} X_{B}\right)^{-1},
\end{aligned}
$$

where $\rho$ is number (or molar) density. The density- and temperature-dependent association strength, $\Delta_{i j}$ is within the SAFT approach given as two-body integral

$$
\Delta_{i j}=\int\left[\exp \left(\frac{u_{i j}(1,2)}{k_{b} T}\right)-1\right] g_{i j}(1,2) d 1 d 2,
$$

where $u_{i j}(1,2)$ is the association energy and $g_{i j}(1,2)$ is the pair distribution function between the associating group in reference system (i.e., when the association is switched off). In practice-oriented equations of state which use the SAFT formalism, the association strength is approximated using two parameters - association volume, $\kappa_{i j}$, and association energy, $\epsilon_{i j}$,

$$
\Delta_{i j}=\kappa_{i j} \beta g_{i j}\left(\sigma_{i j}\right)\left[\exp \left(\frac{\epsilon_{i j}}{k_{b} T}\right)-1\right],
$$

where $\beta$ is some measure of molecular volume and $g_{i j}\left(\sigma_{i j}\right)$ stands for the contact value of the radial distribution function in the reference system. The association energy between sites 
of the same type is zero which leads to $\Delta_{i j}=0$ and to elimination of the respective terms from the above set of equations (the set of three equations is then reduced to one).

The independence of association degree on the chain length for longer alcohols could abet to suggestion that the product $\left(\rho \Delta_{i j}\right)$ is also independent of the chain length. In simplest view, this implies equality of the parameter $\epsilon$ and of the product $\rho \kappa$ for all higher alcohols (with respect to decreasing molar density in homological series this would mean increasing value of associating volume $\kappa$ which is opposite tendency than is usually observed)..$^{39,40,75,76}$

However, the situation is more complicated because of the implicit density dependence of the association strength due to the contact value of rdf, $g_{i j}\left(\sigma_{i j}\right)$. Using the alcohol parameters of the PCSAFT EoS, ${ }^{75}$ we estimated the values of the packing densities (and corresponding contact values of rdf) for all simulated systems and used these values to obtain the parameters $\epsilon$ and $\kappa$ from the temperature dependence of associations strengths. We note that this approach is absolutely inconsistent, since any change of two associating parameters will cause also change the remaining three parameters of PCSAFT (or will lead to incorrect thermodynamic description); a consistent procedure for evaluation of associating parameters from simulation data was suggested by Ferrando et al. ${ }^{63}$

The values of association volume and energy are listed in Table V. The values of both, the association energy $\epsilon$ as well as association volume $\kappa$, are of the correct order of magnitude and they also follow similar trends - increase of the energy and decay of the volume - as do the values for real compounds. The values of associating volume are surprisingly close to the values of Ferrando et al. ${ }^{63}$ (despite we used different force-fields, different HB criteria, different versions of SAFT equation of state, and different approximations in the process of evaluation of these parameters); significantly smaller values of the association energy are consequence of neglected long-range corrections for Lennard-Jones interactions and therefrom higher temperature expansion along coexistence.

The experimental or simulation information about the hydrogen bonding at molecular level can be used to estimate the association parameters of SAFT-like equations of state. ${ }^{63,77}$ At one side, this is a reasonable approach, since the parameters of such equations of state are rather degenerated and the standard optimization on the pure component equilibrium data only can provide several different parameter sets. On the

TABLE V. The association strengths, energy, and volume extracted from the simulated cluster size distribution.

\begin{tabular}{|c|c|c|c|c|c|c|}
\hline \multirow[b]{2}{*}{ Alcohol } & \multicolumn{3}{|c|}{$\Delta_{A C}$} & \multirow[b]{2}{*}{$\epsilon / k_{b}[\mathrm{~K}]$} & \multirow[b]{2}{*}{$\kappa$} & \multirow[b]{2}{*}{$R^{2}$} \\
\hline & $T=300 \mathrm{~K}$ & $T=350 \mathrm{~K}$ & $T=400 \mathrm{~K}$ & & & \\
\hline Ethanol & 0.2102 & 0.1056 & 0.05870 & 769.1 & 0.0855 & 0.9895 \\
\hline Propanol & 0.1748 & 0.0779 & 0.04116 & 1116.0 & 0.0216 & 0.9971 \\
\hline Butanol & 0.2332 & 0.1046 & 0.04986 & 1208.5 & 0.0226 & 0.9995 \\
\hline Hexanol & 0.3092 & 0.1295 & 0.06133 & 1413.7 & 0.0173 & 0.9998 \\
\hline Octanol & 0.3954 & 0.1645 & 0.07202 & 1490.8 & 0.0148 & 0.9973 \\
\hline
\end{tabular}

other hand, since the model is not able to describe correctly the structure at molecular level, the associating parameters may depend on the choice of simulated property taken into account. We consider the optimization of association parameters to equilibrium data of binary mixtures as more suitable way to discriminate between different parameter sets. We also mention, that asymmetric three-site association scheme can improve the description of cluster topology by the 3B SAFT approach, but this would be a step towards larger complexity.

\section{CONCLUSIONS}

The hydrogen bonding in liquid primary alcohols was studied using molecular dynamics simulations. In accordance with previous studies it was found that the structures formed by associated molecules are not linear but they form also treelike branched structures. Small part of molecules participate also on the formation of cyclic structures, typically containing 3-6 molecules. The extent of branching in tree-like aggregates is smaller than it would correspond to the 3B model of SAFT which assumes two independent acceptor sites (and one donor site) per hydroxyl group. Expectably, the average size of associated clusters decays with increasing temperature. On other hand the relative fraction of branching points becomes higher with increasing temperature.

Despite we have simulated alcohols from ethanol to octanol, several important microscopic properties seem to be independent on the size of the chain - the coordination numbers, fraction of non-bonded sites, or association strength (with the exception of ethanol). The resulting parameters describing the association within the SAFT approach are on the other hand dependent on molecular size. A question thus arises, whether and how one can reflect this effect in group-contribution versions of equations of state.

\section{ACKNOWLEDGMENTS}

The authors would like to thank Dr. Moussa Dicko (LSPM) and Dr. Christophe Coquelet (ENSMP) for fruitful discussions. The authors thank the financial support of the French Research National Agency (ANR-09-CP2D-10-03). The access to the MetaCentrum computing facilities provided under the research intent MSM6383917201 is (highly) appreciated.

\footnotetext{
${ }^{1}$ A. Apelblat, J. Mol. Liq. 128, 1 (2006).

${ }^{2}$ H. Kleeberg, D. Klein, and W. A. P. Luck, J. Phys. Chem. 91, 3200 (1987).

${ }^{3}$ H. Graener, T. Q. Ye, and A. Laubereau, J. Chem. Phys. 90, 3413 (1989).

${ }^{4}$ F. Schwager, E. Marand, and R. M. Davis, J. Phys. Chem. 100, 19268 (1996).

${ }^{5}$ S. Woutersen, U. Emmerichs, and H. J. Bakker, J. Chem. Phys. 107, 1483 (1997).

${ }^{6}$ N. Asprion, H. Hasse, and G. Maurer, Fluid Phase Equilib. 186, 1 (2001).

${ }^{7}$ M. Paolantoni, P. Sassi, A. Morresi, and R. Cataliotti, Chem. Phys. 310, 169 (2005).

${ }^{8}$ F. Palombo, P. Sassi, M. Paolantoni, A. Morresi, and R. S. Cataliotti, J. Phys. Chem. B 110, 18017 (2006).

${ }^{9}$ T. Yamaguchi, K. Hidaka, and A. K. Soper, Mol. Phys. 96, 1159 (1999).

${ }^{10}$ I. Bakó, P. Jedlovszky, and G. Pálinkás, J. Mol. Liq. 87, 243 (2000).

${ }^{11}$ M. Tomšič, A. Jamnik, G. Fritz-Popovski, O. Glatter, and L. Vlček, J. Phys. Chem. B 111, 1738 (2007).
} 
${ }^{12}$ J. Towey, A. Soper, and L. Dougan, Phys. Chem. Chem. Phys. 13, 9397 (2011).

${ }^{13}$ P. Sillrén, J. Swenson, J. Mattsson, D. Bowron, and A. Matic, J. Chem. Phys. 138, 214501 (2013).

${ }^{14}$ P. Petong, R. Pottel, and U. Kaatze, J. Phys. Chem. A 103, 6114 (1999).

${ }^{15} \mathrm{~S}$. Schwerdtfeger, F. Köhler, R. Pottel, and U. Kaatze, J. Chem. Phys. 115, 4186 (2001).

${ }^{16}$ P. Huyskens, M. Haulait-Pirson, G. Siegel, and F. Kapuku, J. Phys. Chem. 92, 6841 (1988).

${ }^{17}$ V. A. Durov, Pure Appl. Chem. 76, 1 (2004).

${ }^{18}$ A. Grenner, I. Tsivintzelis, I. Economou, C. Panayiotou, and G. Kontogeorgis, Ind. Eng. Chem. Res. 47, 5636 (2008).

${ }^{19}$ M. Naoki and T. Seki, Fluid Phase Equilib. 281, 172 (2009).

${ }^{20}$ I. Sedov and B. Solomonov, J. Mol. Liq. 167, 47 (2012).

${ }^{21}$ A. Staib, J. Chem. Phys. 108, 4554 (1998).

${ }^{22}$ A. Sum and S. Sandler, J. Phys. Chem. A 104, 1121 (2000).

${ }^{23}$ J.-W. Handgraaf, T. van Erp, and E. Meijer, Chem. Phys. Lett. 367, 617 (2003).

${ }^{24}$ R. Polly, B. Schimmelpfennig, M. Florsheimer, K. Kruse, A. AbdElMonem, R. Klenze, G. Rauhut, and T. Fanghanel, J. Chem. Phys. 130, 064702 (2009).

${ }^{25}$ W. Jorgensen, J. Phys. Chem. 90, 1276 (1986).

${ }^{26}$ M. Haughney, M. Ferrario, and I. McDonald, J. Phys. Chem. 91, 4934 (1987).

${ }^{27}$ J. Padró, L. Saiz, and E. Guàrdia, J. Mol. Struct. 416, 243 (1997).

${ }^{28}$ L. Saiz, J. Padró, and E. Guàrdia, J. Phys. Chem. B 101, 78 (1997).

${ }^{29}$ B. Chen, J. Potoff, and J. Siepmann, J. Phys. Chem. B 105, 3093 (2001).

${ }^{30}$ E. Guàrdia, J. Martí, J. Padró, L. Saiz, and A. V. Komolkin, J. Mol. Liq. 96-97, 3 (2002).

${ }^{31}$ Y. Zhang, J. Yang, Y.-X. Yu, and Y.-G. Li, J. Supercrit. Fluids 36, 145 (2005).

${ }^{32}$ D. Dellis, M. Chalaris, and J. Samios, J. Phys. Chem. B 109, 18575 (2005).

${ }^{33}$ P. Sillrén, J. Bielecki, J. Mattsson, L. Börjesson, and A. Matic, J. Chem. Phys. 136, 094514 (2012).

${ }^{34}$ E. A. Müller and K. E. Gubbins, "Associating fluids and fluid mixtures," in Equations of State for Fluids and Fluid Mixtures, edited by J. Sengers, R. Kayser, C. Peters, and H. J. White (Elsevier, 2000).

${ }^{35}$ M. Wertheim, J. Stat. Phys. 35, 19 (1984).

${ }^{36}$ M. Wertheim, J. Stat. Phys. 35, 35 (1984).

${ }^{37}$ M. Wertheim, J. Stat. Phys. 42, 459 (1986).

${ }^{38}$ M. Wertheim, J. Stat. Phys. 42, 477 (1986).

${ }^{39}$ S. Huang and M. Radosz, Ind. Eng. Chem. Res. 29, 2284 (1990).

${ }^{40}$ S. Huang and M. Radosz, Ind. Eng. Chem. Res. 30, 1994 (1991).

${ }^{41}$ W. Chapman, K. Gubbins, G. Jackson, and M. Radosz, Fluid Phase Equilib. 52, 31 (1989).

${ }^{42}$ W. Chapman, K. Gubbins, G. Jackson, and M. Radosz, Ind. Eng. Chem. Res. 29, 1709 (1990).

${ }^{43}$ G. Kontogeorgis and G. Folas, Thermodynamic Models for Industrial Applications. From Classical and Advanced Mixing Rules to Association Theories (John Wiley and Sons, Ltd., Chichester, 2010).

${ }^{44}$ C. Joslin, C. Gray, W. Chapman, and K. Gubbins, Mol. Phys. 62, 843 (1987).
${ }^{45}$ G. Jackson, W. Chapman, and K. Gubbins, Mol. Phys. 65, 1 (1988).

${ }^{46}$ W. Chapman, G. Jackson, and K. Gubbins, Mol. Phys. 65, 1057 (1988).

${ }^{47}$ R. Sear and G. Jackson, Phys. Rev. E 50, 386 (1994).

${ }^{48}$ R. Sear and G. Jackson, Mol. Phys. 82, 1033 (1994).

${ }^{49}$ R. Sear and G. Jackson, J. Chem. Phys. 105, 1113 (1996).

${ }^{50} \mathrm{~S}$. Derawi, J. Zeuthen, M. Michelsen, E. Stenby, and G. Kontogeorgis, Fluid Phase Equilib. 225, 107 (2004).

${ }^{51}$ J. Janeček and P. Paricaud, J. Phys. Chem. B 116, 7874 (2012).

${ }^{52}$ J. Janeček and P. Paricaud, J. Phys. Chem. B 117, 9430 (2013).

${ }^{53} \mathrm{G}$. W. Huber, S. Iborra, and A. Corma, Chem. Rev. 106, 4044 (2006).

${ }^{54}$ N. Mac Dowell, F. Pereira, F. Llovell, F. Bias, C. Adjiman, G. Jackson, and A. Galindo, J. Phys. Chem. B 115, 8155 (2011).

${ }^{55}$ J. Rodriguez, N. Mac Dowell, F. Llovell, C. Adjiman, G. Jackson, and A. Galindo, Mol. Phys. 110, 1325 (2012).

${ }^{56} \mathrm{~S}$. Tamouza, J.-P. Passarello, P. Tobaly, and J.-C. de Hemptinne, Fluid Phase Equilib. 222-223, 67 (2004).

${ }^{57}$ S. Tamouza, J.-P. Passarello, P. Tobaly, and J.-C. de Hemptinne, Fluid Phase Equilib. 228-229, 409 (2005).

${ }^{58}$ A. Lymperiadis, C. Adjiman, A. Galindo, and G. Jackson, J. Chem. Phys. 127, 234903 (2007).

${ }^{59}$ A. Lymperiadis, C. Adjiman, G. Jackson, and A. Galindo, Fluid Phase Equilib. 274, 85 (2008).

${ }^{60}$ D. NguyenHuynh, J.-P. Passarello, P. Tobaly, and J.-C. de Hemptinne, Fluid Phase Equilib. 264, 62 (2008).

${ }^{61}$ D. NguyenHuynh, A. Falaix, J.-P. Passarello, P. Tobaly, and J.-C. de Hemptinne, Fluid Phase Equilib. 264, 184 (2008).

${ }^{62}$ D. NguyenHuynh, J.-P. Passarello, J.-C. de Hemptinne, and P. Tobaly, Fluid Phase Equilib. 307, 142 (2011).

${ }^{63}$ N. Ferrando, J.-C. de Hemptinne, P. Mougin, and J.-P. Passarello, J. Phys. Chem. Phys. B 116, 367 (2012).

${ }^{64}$ J. Ponder, TINKER: Software Tools for Molecular Design (Washington University, St. Louis, MO, 2011).

${ }^{65}$ H. Berendsen, J. Postma, W. van Gunsteren, A. DiNola, and J. Haak, J. Chem. Phys. 81, 3684 (1984).

${ }^{66}$ W. Jorgensen, D. Maxwell, and J. Tirado-Rives, J. Am. Chem. Soc. 118, 11225 (1996).

${ }^{67}$ A. Luzar and D. Chandler, J. Chem. Phys. 98, 8160 (1993).

${ }^{68}$ L. Saiz, E. Guardia, and J. Padro, J. Chem. Phys. 113, 2814 (2000).

${ }^{69}$ Prosim. Component Plus database (based on DIPPR), 3.8.0.1 ed., 2001.

${ }^{70}$ A. Trokhymchuk and J. Alejandre, J. Chem. Phys. 111, 8510 (1999).

${ }^{71}$ J. Janeček, H. Krienke, and G. Schmeer, J. Phys. Chem. B 110, 6916 (2006).

${ }^{72}$ J. Janeček, H. Krienke, and G. Schmeer, Condens. Matter Phys. 10, 415 (2007).

${ }^{73}$ J. Janeček, J. Chem. Phys. 131, 124513 (2009).

${ }^{74}$ J. Janeček, Oil Gas Sci. Technol. 68, 271 (2013).

${ }^{75}$ J. Gross and G. Sadowski, Ind. Eng. Chem. Res. 41, 5510 (2002).

${ }^{76}$ G. Kontogeorgis, M. Michelsen, G. Folas, S. Derawi, N. von Solms, and E. Stenby, Ind. Eng. Chem. Res. 45, 4855 (2006).

${ }^{77}$ G. Clark, A. Haslam, A. Galindo, and G. Jackson, Mol. Phys. 104, 3561 (2006) 